\title{
Construction and Optimization of Virtual Marine Engine Room with Hybrid Modeling Technology Based on Geometry and Image
}

\author{
DAI Yuliang ${ }^{1,}$, KONG Qingfu $^{1}$, WANG Guosong ${ }^{2}$, SONG Fei ${ }^{1}$ \\ ${ }^{1}$ Scientific Research Department, Naval University of Engineering, Wuhan 430033, Hubei, China \\ ${ }^{2}$ Center of Equipment Repair and Surveillance, Eastern Sea Naval Fleet, Shanghai 200000, China \\ ayuliang_dai@163.com
}

Keywords: Virtual marine engine room, Hybrid modeling technology based on geometry and image, Projection matching, Detail hierarchy model

\begin{abstract}
It is usually difficult to construct the virtual scene of complex environment such as the ship engine room. Therefore, a new method for virtual engine room construction using projection matching and hybrid modeling technology based on geometry and image is presented in this paper. In this method, the optimization technology based on the combination of substitution, bulletin board and detail hierarchy model is adopted so that both a good interactive feature of the virtual cabin can be achieved and the workload can be reduced. Meanwhile, the fidelity and truthfulness degree are all improved. It is proved that a better result can be got with the method.
\end{abstract}

\section{Introduction}

The marine engine room is not only the place to set the powerplant but also the main working environment of electromechanical managers. Virtul marine engine room is an important part of the virtual training system of the marine powerplant. With the help of a virtual engine room environment with excellent fidelity and interactivity characteristics, the existence feelings of trainees can be significantly enhanced, and the training effect can be effectively improved. However, the internal pipelines in the marine engine room are multifarious with numerous instruments, and their geometric models are very complex. Thus it is usually a huge project to construct the virtual engine room with the traditional 3D modeling technology. Therefore, a new method for fast reconstruction of the virtual engine room using hybrid modeling technology based on geometry and image is proposed in order to satisfy the requirements of usage and operation training of marine engines in this paper.

\section{Construction of Virtual Marine Engine Room with Hybrid Modeling Technology Based on Geometry and Image}

Hybrid Modeling Technology Based on Geometry and Image. Hybrid rendering is the technology using both geometry and image at the same time as the basic element to draw the picture[1]. According to a certain standards, parts of scenes are dynamically simplified as texture images mapped on simple geometries. If the error resulted by simplification is less than the given threshold, the texture images are then directly used to replace the original scenes and draw the pictures. Simple geometric surface should be set at the center of simplified scenery, and the simplification error should be strictly controlled within a given threshold. Under a certain error conditions, scene images can be rapidly produced with low cost while using this drawing technology. Meanwhile, right order and high quality pictures can be also achieved.

The so-called modeling technology based on geometry and image, refers to that the whole 3D structure is built using 3D entity, and some complex details or environment backgrounds are constructed with images, so that a realistic and quickly rendering virtual reality scene with moderate amount of data can be established. It mainly includes two aspects: (1) As to the scene drawing, drawing methods based on both the geometry and the image are combined together, images are used dynamically to replace some geometric objects while the scene drawing. Therefore, triangle 
amounts needed by actual drawing can be reduced and the scene rendering speed can be accelerated; (2) As to the scene modeling, basic geometric shapes of complex objects are constructed at first while modeling complex objects in the scene. Then, the texture mapping is adopted to enhance the fidelity of the object's model. With this method, amount of the triangles included in complex object and complexity of the virtual reality scene rendering can be reduced effectively.

Therefore, the key point of hybrid modeling technology is to combine the 3D model and 2D image organically. The following problems should be solved firstly:

(1)Visual consistency. Entities in virtual environment should be visually consistent with integrated images during both static observed or roamed in the virtual environment. That is to say, the consistency of perspective relation and spatial relation should be kept. For example, size and view angle of 3D models in a virtual environment should be consistent with the virtual environment based on images while users moving forward or backward.

(2) Motion consistency. size and view angle of 3D objects' models in a virtual environment should be consistent with the virtual environment based on images while the $3 \mathrm{D}$ models being moved in the virtual environment, such as parallel movement or rotation.

(3) Illumination consistency. Illumination effect of 3D models, such as shadow and highlight, should be consistent with the virtual environment based on images.

As to the virtual engine room to be constructed in this study, key point of the problems mentioned above is to keep the visual consistency. First of all, the engine room is a closed environment without natural light interference. Positions and lighting degrees of lights in the engine room are normally fixed. The lights are usually turned on and lighting environment is normally simple. Thus, requirement on illumination consistency is relatively not so strong. Secondly, movements of objects inside the engine room are relatively simple. Main equipment are always set in fixed positions and movements usually occur inside the equipment. As to the virtual engine room used for scene roaming and operational training, detail moving courses of many equipment are taken place without person-interference. Therefore, modeling work of illumination consistency and movement consistency can be largely reduced.

Visual consistency method based on projection matching. The virtual engine room environment based on image is a 2D plane which some equipment in the engine rom are projected on. The basic principle of perspective projection is shown in Fig. 1 where the projection plane, namely the virtual engine room environment, includes the projection image of the 3D model 1 , namely equipment in the engine room. The key point to keep the visual consistency between the 3D model 1 and the virtual engine room environment is to calculate the location and parameters of the actual camera accurately and to determine a correct cover relation between the 3D model 1 and the virtual engine room environment so that the 3D model 1 is placed into a proper position in the virtual engine room with proper size[2]. The visual consistency can not be kept because the position of view point is changed while users roaming in the virtual environment. So virtual cameras are needed to be re-calibrated in order to keep the visual consistency between the $3 \mathrm{D}$ virtual objects and the scene, otherwise the position of 3D virtual objects must be changed with the motion of view point. A novel method based on the basic principle of perspective projection for keeping the visual consistency between the 3D model and the 2D image by changing the 3D model's position, orientation and attitude in this study, namely projection matching method, as shown in Fig.2.

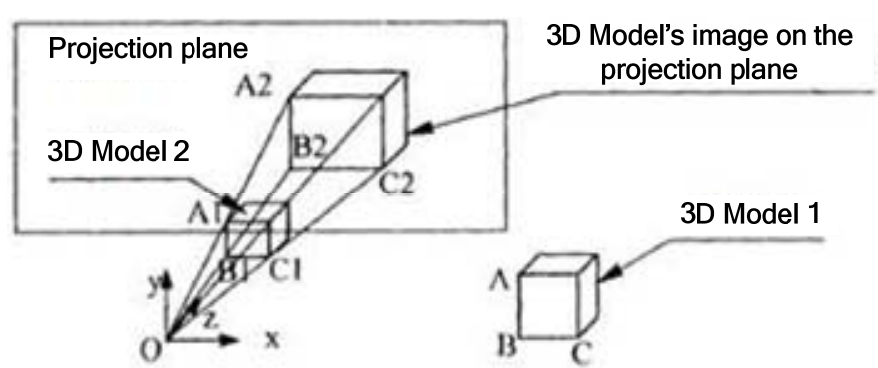

Fig.1 The Geometric Principle of projection

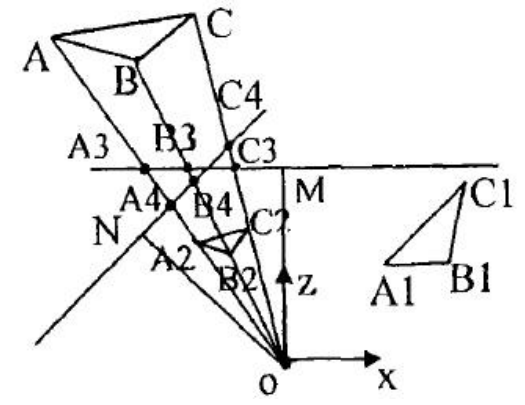

Fig. 2 The Geometric Principle of Projection Matching in a plane 
In fig. 1 , the 3D model 2 is a imaginary copy of the 3D model 1, and the visual consistency between the 3D object and the projection plane will be kept if the 3D object moves to the position of model 2 from the position of model 1 by translation or rotation.According to the principle of perspective projection[3], namely the similar triangles principle, the projection points A2, B2, C2 of three points A1, B1, C1 on the 3D model 2 corresponding to the points $\mathrm{A}, \mathrm{B}, \mathrm{C}$ chosen on the 3D model 1 are sure to be found in the projection plane respectively, and vice versa. Therefore the coordinates of the points A1, B1, C1 will be obtained if the coordinates of the projection points A2, B2, C2 are known. Then the 3D model 1 is moved to the position of the 3D model 2 by translation and rotation so that $\mathrm{A}, \mathrm{B}, \mathrm{C}$ coincide with A1, B1, C1. The information of the 3D object's translation and rotation is described by quaternion.

In fig.2, $\mathrm{ABC}$ is an actual object in the engine cabin and $\mathrm{A} 1 \mathrm{~B} 1 \mathrm{C} 1$ is its corresponding $3 \mathrm{D}$ model in the virtual engine rom. When view direction is $\mathrm{OM}$, the equipment $\mathrm{ABC}$ in the actual scene is projected onto the plane and the image A3B3C3 in the projection plane is obtained. A2B2C2 is an imaginary object of A1B1C1. According to the principle of perspective projection, it is easy to obtain the coordinates of the points A2, B2, C2 satisfying the visual consistency condition. When the view direction is changed, such as the view direction turning to the direction $\mathrm{ON}$, the projection point $\mathrm{A} 3, \mathrm{~B} 3, \mathrm{C} 3$ of actual device are fixed, but the projection image of the 3D model A2B2C2 in the new view direction is A4B4C4. Apparently the points A3, B3, C3 coincide with the points A4, $\mathrm{B} 4, \mathrm{C} 4$ respectively. In other words, the visual consistency between A2B2C2 and the projection image of the actual object is still kept when the location of viewpoint is changed. It is easy to determine the position of the points A3, B3, C3 in the two-dimensional coordinate system because the depth information of the image is omitted. The position of A2B2C2 is fixed. The Quaternion method is used to make the points A1, B1, C1 coincide with the points A2, B2, C2 so that the visual consistency relationship between the 2D image and 3D model is satisfied.

\section{Scene optimization technology based on substitutions and bulletin boards}

Scene optimization will aim to make the scene to reduce the demand of hardware and Improve rendering speed by Model simplification and image rendering without affecting the visual effect. In addition, the special rendering method is used to enhance the fidelity and immersion. In this paper, the image rendering technology based on substitutions is applied to optimization of virtual scene. That is to say,the 3D model can be replaced by the geometry with its surface attached by texture image so as tosimplify the scene and enhance the reality.

Texture Mapping. Texture refers to the surface details of object. The surface detail of object is generally divided into two types as follows: one is the color texture, such as a variety of two-dimensional pattern on the vase; the other is a geometric texture, such as wrinkles on orange surface. Texture mapping method may be used to add an image onto the surface of object. The general method for creating color texture as follow: at first, texture pattern is defined in a flat area, namely texture space, then the corresponding relation between the points on the object's surface and texture space, namely mapping, is established. After the visible points on object's surface are confirmed, the texture pattern can be attached to the surface of the object with the value of the corresponding point on texture space multiplying by the brightness value. Similar methods can also be used to produce uneven surface appearance, named bump texture. But here the texture values act on the normal vector, rather than on the color brightness. Whether the color texture or bump texture is similar to the actual object looked with no accurate simulation so that the amount of calculation is not significantly increased and the reality is greatly enhanced.

Texture mapping technology is widely used to enhance the reality of entity surface in the computer graphics area. It is also an effective way to control scene complexity and accelerate graphics rendering. The principle of texture mapping is either mapping a two-dimensional texture image to the three-dimensional surface of entity, or modifying the light intensity distribution on the surface of entity.For example, the texture image such as a pattern or photo of floor is mapped to a rectangular plane in order to create a model of wooden floor. Thus, more triangles isn't needed to represent each stripe on the wooden floor, and also the details of the wooden floor can be 
realistically described. Texture mapping is performed between the image space, object space, and the texture space as shown in Fig.3.

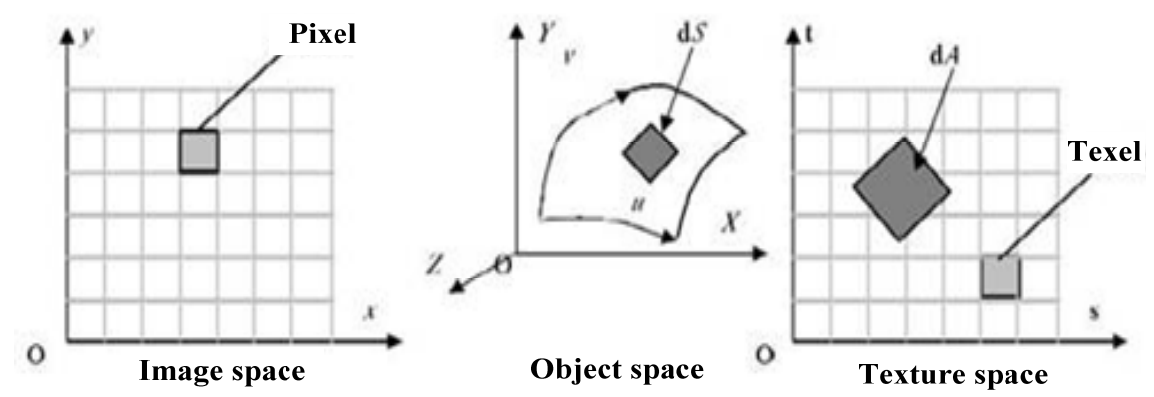

Fig. 3 The steps of Texture Mapping

The steps of Texture Mapping are as follows: At first, a pixel region on the screen is mapped to the surface of the object, namely, an image space is mapped to an object space; Secondly, the curved surface region dS corresponding to the pixel on the surface of object is mapped to texture area $\mathrm{dA}$ in the texture space, that is to say, an object space is mapped to an texture space.

Hybrid rendering method of virtual engine room based on the combination of substitution and detail hierarchy model. The so-called substitution refers to the simple geometric object which is the replacement of object with complex geometric properties. The substitution technology is a rendering technique based

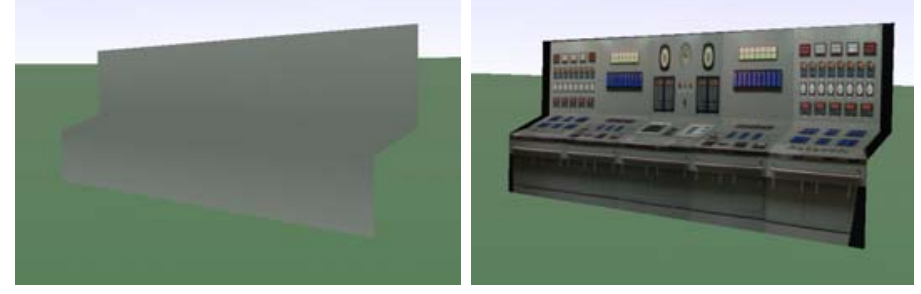

Fig.4 An example of impostor on image, namely, the object with complex geometric properties is replaced by an simple geometric object so as to reduce the geometric complexity of the objects in the scene and improve the rendering efficiency of the scene. As shown in Fig.4, there are a simple geometry whose shape is similar to the console on the left and a console who is obtained from the left geometry attached by the photo texture of the console on the right. Maciel, etc.[4] first proposed that the substitution technology was used to accelerate rendering. Their idea is that the geometric model of the object is replaced by a two-dimensional bulletin board attached by the photo texture of the object. The cost of calculation for producing and rendering a substitution is very small, so the substitution adapts to being generated dynamically. However, when the viewpoint moves, the parallax effect will be very obvious if the substitution isn't updated in time. Therefore it is necessary to check the effectiveness of the substitution. Schaufler, etc.[5,6] put forward that the substitution didn't need to be updated if only the view direction was changed and the substitution might need to be updated while the viewpoint moved. A calculation formula of the parallax is needed to obtain the Range of viewpoint movement within which the substitution didn't need to be updated. Jakulin simply regards the parallax as the angle between the direction from the current viewpoint to the substitution and the view direction in time of the substitution generation[7].

In this study, the combination of substitution and detail hierarchy model is adopted to model the equipment in the engine room. A different level of detail is selected in the different subregion of the scene, and the same modeling method is used to model the equipment in the same level region. Thus it is avoided that the hierarchical levels of detail is calculated individually for each model of the equipment in the scene, and the time of the entity detail levels optimization in the scene is reduced. On the other hand, only a few models of the important equipment who is close to the viewpoint are the full geometric model, and the others are image-based model. Hence, the number of triangles who need to be rendered greatly decrease, and the complexity of the whole scene rendering is reduced. 


\section{Conclusions}

In this paper, the hybrid modeling technology based on geometry and image is used to construct virtual marine engine room. The optimization technology based on the combination of substitution, bulletin board and detail hierarchy model is adopted so that both a good interactive feature of the virtual cabin can be achieved and the workload can be reduced. Meanwhile, the fidelity and truthfulness are all improved. It is proved that a better result can be got using this method. Also, the procedure can offer a valuable reference for similar complex virtual environments construction.

\section{References}

[1] Zhang hanling, Hao chongyang, Fan yangyu. Hybrid Rendering Approach Based Image and Graphics. Computer Engineering and Applications, No.8(2003), P.101-104.

[2] Gao yu, Song hanzhen, Wu lingda. Virtual Objects Registration in Imaging Space. Computer Engineering and Applications, No.30(2003), P.47-50.

[3] Zhang shunxin,Ye huan. Projection and Painting Art. Journal of Engineering Graphics, No.5(2008), P.107-110.

[4] Maciel P W C, Shirley P. Visual navigation of large environments using textured clusters. Proc of Symposium on Interactive 3D Graphics Monterey, California ACM Press(1995), P.95-102.

[5] Schaufler G . Dynamically generated impostors . Fellner P W . Modeling-virtual worlds-distributed graphics. Proc of MVD’95 Workshop Gemany, Infix Verlag, 1995: 129-135.

[6] Schaufler G, Sturzlinger W. A three dimensional image cache for virtual reality. Computer Graphics Forum, Vol.15, N0.3(1996), P.227.

[7] Jakulin A. Interactive vegetation rendering with slicing and blending. Proc of Eurographics Interlaken(2000), P.96-100. 